\title{
Correction of susceptibility artifacts in diffusion tensor data using non-linear registration
}

\author{
D. Merhof ${ }^{a, b, *}$, G. Soza ${ }^{c}$, A. Stadlbauer ${ }^{b}$, G. Greiner ${ }^{a}$, \\ C. Nimsky ${ }^{b}$ \\ ${ }^{a}$ Computer Graphics Group, University of Erlangen-Nuremberg, Am \\ Weichselgarten 9, 91058 Erlangen, Germany \\ ${ }^{\mathrm{b}}$ Dept. of Neurosurgery, University of Erlangen-Nuremberg, Schwabachanlage 6, \\ 91054 Erlangen, Germany \\ cSiemens Medical Solutions, Siemensstr. 1, 91301 Forchheim, Germany
}

\begin{abstract}
Diffusion tensor imaging can be used to localize major white matter tracts within the human brain. For surgery of tumors near eloquent brain areas such as the pyramidal tract this information is of importance to achieve an optimal resection while avoiding post-operative neurological deficits. However, due to the small bandwidth of echo planar imaging, diffusion tensor images suffer from susceptibility artifacts resulting in positional shifts and distortion. As a consequence, the fiber tracts computed from echo planar imaging data are spatially distorted. We present an approach based on non-linear registration using Bézier functions to efficiently correct distortions due to susceptibility artifacts. The approach makes extensive use of graphics hardware to accelerate the non-linear registration procedure. An improvement presented in this paper is a more robust and efficient optimization strategy based on simultaneous perturbation stochastic approximation (SPSA).

Since the accuracy of non-linear registration is crucial for the value of the presented correction method, two techniques were applied in order to prove the quality of the proposed framework. First, the registration accuracy was evaluated by recovering a known transformation with non-linear registration. Second, landmark-based evaluation of the registration method for anatomical and diffusion tensor data was performed. The registration was then applied to patients with lesions adjacent to the pyramidal tract in order to compensate for susceptibility artifacts. The effect of the correction on the pyramidal tract was then quantified by measuring the position of the tract before and after registration. As a result, the distortions observed in phase encoding direction were most prominent at the cortex and the brainstem. The presented approach allows correcting fiber tract distortions which is an important prerequisite when tractography data are integrated into a stereotactic setup for intra-operative guidance.
\end{abstract}


Key words: susceptibility artifacts, diffusion tensor imaging, non-linear registration, graphics hardware, neuro-navigation

\section{Introduction}

Diffusion tensor imaging (DTI) is a non-invasive in vivo imaging modality which is able to differentiate between white and grey matter within the human brain. It is based on the phenomenon of water diffusion which is associated with Brownian motion. Major white matter tracts consist of areas of a strongly aligned microstructure. The diffusion behavior within those areas is anisotropic since the aligned microstructure restricts the diffusion to a preferred direction. DTI measures this effect and thus reflects both tissue structure and architecture at the microscopic level (Basser et al., 1994). For this purpose, at least seven images are acquired: A reference image measured without diffusion sensitization, and six diffusion-weighted (DW) images acquired with diffusion gradients in non-collinear directions. Based on this series of directional diffusion images, the location of major white matter tracts is obtained by computing tensors which are either visualized using scalar metrics (Basser and Pierpaoli, 1996; LeBihan et al., 2001; Westin et al., 2002), glyph representations (Kindlmann, 2004) or techniques commonly referred to as tractography (Mori et al., 1999; Basser et al., 2000; Mori and van Zijl, 2002).

As a drawback, DTI measurements based on single-shot echo planar imaging (EPI) suffer from image distortions, primarily eddy current induced image distortions, chemical shift, and susceptibility artifacts. These artifacts are a severe problem if fiber tracts reconstructed from EPI data are integrated into anatomical data, e.g. acquired by a 3D gradient echo sequence, for functional neuro-navigation. In recent years, integration of pre-operative functional data from functional MRI (fMRI) or magnetoencephalography (MEG) into anatomical data, known as functional neuro-navigation, has become routine in surgery of lesions adjacent to eloquent cortical brain areas, such as the motor strip or language-related areas, avoiding post-operative neurological deficits (Ganslandt et al., 2004; Kober et al., 2001; Nimsky et al., 1999). Besides eloquent cortical brain areas also deep seated structures, such as major white matter tracts, have to be preserved during surgery. Integration of fiber tracts into anatomical data used for stereotactic guidance, allows visualizing the spatial

* Corresponding author. Tel: +49-9131-8525255 Fax: +49-9131-8525249

Email address: Dorit.Merhof@informatik.uni-erlangen.de (D. Merhof).

$U R L$ : www9.informatik. uni-erlangen.de/people (D. Merhof). 
relation of a tumor and adjacent white matter tracts for neurosurgical planning (Clark et al., 2003; Coenen et al., 2001; Kamada et al., 2005; Kinoshita et al., 2005). However, an important prerequisite for the use of fiber tract information in a stereotactic coordinate system is to correct the distortions of the DTI data. Otherwise, the spatial relation between anatomy depicted by standard MR such as T1- or T2-weighted data and the fiber tract data would be incorrect. In contrast to EPI, the distortion of anatomical data is negligible in a clinical setting and accordingly they are routinely used for neuro-navigational guidance (Ganslandt et al., 2004; Nimsky et al., 2004a; Nimsky et al., 1999). Correcting the different types of EPI distortions has been partly addressed either by enhanced acquisition schemes or by post-processing steps.

Eddy currents are induced by the switching of the diffusion gradients in DW pulse sequences. They cause a spatial misalignment of the DW volumes and are a common problem in clinical DW image acquisitions. For this reason, gradient preemphasis schemes are currently implemented in most MRI scanners to effectively reduce the impact of eddy currents. Alternatively, post-acquisition strategies (Rohde et al., 2004; Shen et al., 2004; Mangin et al., 2003; Bodammer et al., 2004) have been presented which do not depend on a properly calibrated gradient preemphasis.

Chemical shift and susceptibility artifacts can be attributed to the small bandwidth of the EPI sequence in phase encoding direction. Differences in chemical shift or susceptibility cause slight differences of the proton resonant frequency resulting in positional shifts and distortions when the positions of the signal are mapped onto the image by frequency. To minimize the image distortions originating from chemical shift, the EPI scans are usually acquired with lipid suppression. For this purpose, a saturating pulse is commonly applied to the fat resonance prior to data acquisition (Weisskoff, 1990). Another promising approach is to combine chemical shift and slice selective excitation (Meyer et al., 1990).

Susceptibility artifacts (Jezzard and Clare, 1999) resulting from microscopic gradients or variations in the magnetic field strength are observed at the interface of tissues with different magnetic susceptibility. They appear at the proximity of the skull base and near other air-filled spaces such as the brainstem and the frontal lobe and cause severe distortions implying a displacement of anatomical structures. First attempts to account for susceptibility artifacts inherent to EPI use non-linear registration schemes. A hierarchical multigrid algorithm estimating a deformation field by minimizing a cost function based on mutual information using Powell's optimization scheme (Press et al., 2002) is proposed by Hellier et al (Hellier and Barillot, 2000). Another approach for correcting EPI distortions uses a higher-order spline model for warping and a similarity measure based on squared differences (Kybic et al., 2000). In their work, Kybicet al encounter the problem of local minima, which they identified 
as a potential source of error in their approach. With Powell's optimization strategy we employed in our previous work, we also encountered this problem resulting in an unsatisfying registration result. For this reason, we present an implementation taking advantage of the SPSA optimization strategy which is explicitly designed to better overcome the problem of local minima.

In this paper, an approach accounting for susceptibility artifacts based on a non-linear registration strategy is proposed which is built on top of our previous work: We introduced hardware accelerated 3D texture mapping for rigid registration (Hastreiter and Ertl, 1998) which is an extension to pure software based approaches (Wells et al., 1995; Collignon et al., 1995; Studholme et al., 1996; Studholme et al., 1999). This technique became more robust and efficient by using pre-segmented brain volumes (Hastreiter et al., 2000) and by the use of hardware-based 3D Bézier functions (Soza et al., 2002). Thereby, graphics hardware is extensively used in order to considerably accelerate the registration procedure. Further improvements were achieved by adaptive refinement of the underlying control point grid (Hastreiter et al., 2004). The registration strategy also proved to be adequate for parameter estimation in brain shift simulation (Soza et al., 2004). Extending our previous results and based on initial experience with non-linear registration of DTI data (Merhof et al., 2004), an improved registration scheme is presented in this work. Essentially, this paper comprises two major contributions: From a technical point of view, the approach for non-linear registration became more robust and was accelerated by using the simultaneous perturbation stochastic approximation (SPSA) (Spall, 1992) optimization strategy. With respect to medical relevance and application, a clinical evaluation is presented where the extent of image distortion was investigated with respect to patients with lesions adjacent to the pyramidal tract. Overall, this work addresses an important prerequisite for the integration of DTI into neuro-navigation and suggests an efficient and robust solution.

The general outline of the paper is as follows: In Section 2, important components of the registration algorithm are presented comprising the similarity measure (2.1), the deformation model (2.2) and the optimization strategy (2.3). The setup of the registration framework based on these components is resumed in Section 2.4. Subsequently, the experimental setup and all evaluation techniques are described in Section 3 including the acquired image sequences (3.1), the approach for evaluating the registration accuracy (3.2) and the experimental setup for distortion correction (3.3). The results presented in Section 4 comprise an evaluation investigating the quality of registration (4.1) as well as the measurement results of the experimental setup (4.1). Finally, the registration approach and its relevance for clinical application are discussed in Section 5. 


\section{Registration}

In order to correct for image distortions due to susceptibility artifacts inherent to EPI data, an intensity-based registration approach was developed. Within this context, a non-linear transformation has to be determined that maps the DTI reference image (B0) onto the anatomical MR image. This kind of registration problem is still a challenge due to the high computational expenses and due to the low resolution of DTI data. The presented approach aims at clinical application which implies rigorous requirements with respect to computation times. To guarantee an alignment of high quality, we employed normalized mutual information as similarity measure (Section 2.1). Concerning the computational costs, an important issue is the choice of the optimization strategy since the number of optimization iterations as well as computation times to determine the global optimum in each iteration are crucial for the overall performance of the registration. For this reason, we applied a highly efficient optimization strategy using simultaneous perturbation stochastic approximation (Section 2.3), which ensures high convergence rates. Another limiting factor for the performance of intensity-based non-linear registration algorithms is the huge amount of interpolation operations needed for repeatedly computing the similarity measure during optimization. This consideration has motivated the application of graphics hardware for registration purposes. A powerful model for 3D free-form deformation (FFD) based on Bézier functions as well as hardware-based techniques enabling an efficient implementation are presented in Section 2.2.

\subsection{Similarity measure}

Although there exists a number of measures potentially suitable to the context of registration, in practice recent image registration algorithms mainly use two similarity measures: the normalized mutual information (NMI) (Studholme et al., 1999) or the correlation ratio (Roche et al., 1998). However, correlationbased methods require a linear dependence between the intensities, which restricts their application with respect to multimodal registration. Since susceptibility distortions are accompanied by a shift of grey values, correlation-based methods are not adequate for this application. Additionally, an appropriate choice for the underlying similarity measure is essential since this measure has to be calculated many times during a registration. Therefore, the computational costs of evaluating this function including the time complexity of one function calculation and the total evaluation time related to the convergence rate achieved with a given similarity measure should be as low as possible.

Currently, the most generally formulated similarity measure is NMI (Studholme 
et al., 1999). This information theoretic function is based on the Shannon entropy (Shannon and Weaver, 1949) $H(I)$ which, in reference to an image, is a measure of the diversity of the intensity distribution. However, the classical image registration problem considers two images involving two intensities at each voxel position which is depicted by the joint entropy $H\left(I_{s}, I_{t}\right)$ where $I_{s}$ denotes the source image and $I_{t}$ the target image. If the joint entropy is considered only, this similarity measure would make the registration very dependent on the overlapping volume of the images. Thus, mutual information (MI) (Collignon et al., 1995; Wells et al., 1997) also incorporates the marginal entropies related to the intensity probability distribution in the overlapping region for each image separately.

However, mutual information is not entirely independent of the overlapping region of two images. Changes in some regions can disproportionately influence the value of the similarity measure. Circumventing this effect, NMI was proposed as

$$
N M I\left(I_{s}, I_{t}\right)=\frac{M I\left(I_{s}, I_{t}\right)}{H\left(I_{s}, I_{t}\right)}+1=\frac{H\left(I_{s}\right)+H\left(I_{t}\right)}{H\left(I_{s}, I_{t}\right)}
$$

where MI is normalized with respect to the joint entropy. This normalized version of MI proved to be considerably better than standard MI and was therefore used in our registration framework.

\subsection{D free-form deformation}

To accomplish non-linear registration, a FFD model based on 3D Bézier functions is used and graphics hardware is employed to accelerate the approach (Soza et al., 2002; Soza, 2005). The main idea of FFD is to warp the space surrounding an object which will be warped implicitly. This type of FFD has a number of advantages: It contains inherent smoothness as well as elasticity, which makes it very suitable for describing the deformation of soft tissue. Moreover, Bézier functions are equipped with a simple mechanism for their modification and are characterized by intuitive behavior on alteration.

As a drawback, high computational cost is necessary for the evaluation of 3D Bézier functions. Especially in the context of registration, many calculations of Bézier functions have to be performed. Therefore, in order to make the method more efficient, graphics hardware was used to accelerate the FFD approach. 


\subsubsection{Deformation model}

In order to describe a deformation utilizing Bézier functions in mathematical terms, the object space $\mathcal{O S}$ is associated with the transformed B0 dataset and is parameterized with the function $\mathbf{P}: \mathcal{P S} \mapsto \mathcal{O S}$ leading from parameter space $\mathcal{P} \mathcal{S}$ being $[0,1]^{3}$ to object space. For the purpose of deformation, the function $\mathbf{D}: \mathcal{P S} \mapsto \mathcal{T} \mathcal{S}$ is introduced which is parameterized over the defined parameter space and leads to the texture space $\mathcal{T} \mathcal{S}$. A lattice consisting of control points $b_{i, j, k}(i=0, \ldots, l, j=0, \ldots, m, k=0, \ldots, n)$ is placed in the texture space and defines the shape of function

$$
\mathbf{D}(s, t, u)=\sum_{i=0}^{l} \sum_{j=0}^{m} \sum_{k=0}^{n} B_{i}^{l}(s) B_{j}^{m}(t) B_{k}^{n}(u) b_{i, j, k}
$$

in a unique way. This function $\mathbf{D}$ is a trivariate tensor product of $1 \mathrm{D}$ Bézier functions where the basis functions $B_{i}^{l}, B_{j}^{m}, B_{k}^{n}$ are Bernstein polynomials of order $l, m$ and $n$, respectively (Figure 1). Changes in the form of the deformation represented by function $\mathbf{D}$ can be achieved with movements of the lattice points $b_{i, j, k}$.

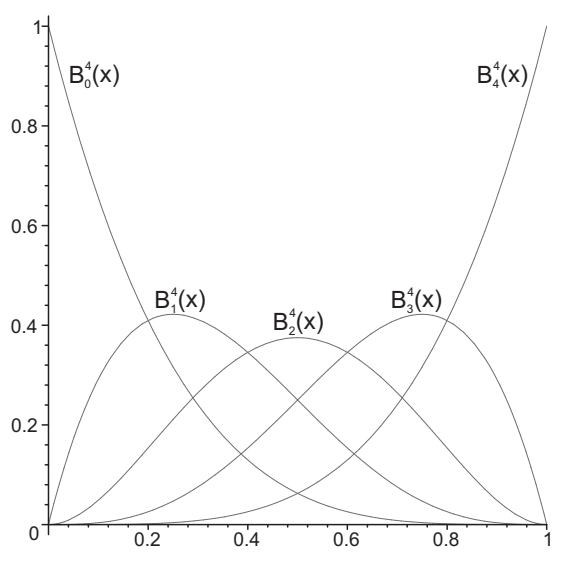

Fig. 1. Bernstein polynomials of order 4

In the context of registration, an important property of any Bézier function is the fact that it lies within the convex hull of the control points: $\mathbf{D}(s, t, u) \in\left\{\sum_{i, j, k} c_{i, j, k} \cdot b_{i, j, k}\right\}$, where $\sum_{i, j, k} c_{i, j, k}=1$ and $i=0, \ldots, l$, $j=0, \ldots, m, k=0, \ldots, n$. This property ensures that the Bézier function smoothly follows the control points without erratic oscillations. This is utilized in the registration algorithm by setting the border control points to be fixed. Therefore, we can prevent the deformation function from taking values from outside of the registered image volume. 


\subsubsection{Hardware-accelerated free-form deformation}

In order to make the FFD model efficient in spite of the high computational cost of evaluating 3D Bézier functions, graphics hardware was used for speedup. For this purpose, the image data is initially loaded into 3D texture memory of the graphics adapter to employ the texture processing unit for performing the most expensive computations. The texture space $\mathcal{T} \mathcal{S}$ being $[0,1]^{3}$ is associated with the texture memory in order to perform texture mapping.

The calculation of a single FFD consists of three steps. In the first step, the object is embedded in the lattice of control points. The control points lie in the texture space, while the object undergoing deformation is physically placed in the texture memory and in a logical sense in the object space. Initially, the control points in the lattice are uniformly displaced in the texture space.

To produce a deformation, in the second step control points are shifted to their new locations. This changes the structure of the control lattice which is expressed by the function

$$
\begin{aligned}
\mathbf{M}: \mathcal{T} \mathcal{S} & \mapsto \mathcal{T} \mathcal{S}, \\
\mathbf{M}\left(b_{x}, b_{y}, b_{z}\right) & =\left(t_{x}, t_{y}, t_{z}\right) .
\end{aligned}
$$

In our method, the changes in the control lattice are not directly based on the absolute coordinates of the control points. Instead, offset vectors $\left(t_{x}, t_{y}, t_{z}\right)$ from the initial control points positions $\left(b_{x}, b_{y}, b_{z}\right)$ are chosen as the free parameters of the Bézier transformation. This strategy allows treating the occurring free-form deformation as a change of a vector field containing an object which is deformed. Such a treatment is closely related to the physical nature of the phenomenon. In the initial stage, the vector field is set to 0 at each control point. In order to prevent illegal values of the deformation function, the offset vectors at the border are set to 0 during the whole registration procedure. Thus, only the inner control points of the Bézier function are free parameters of the optimization. This assumption is well motivated in practice since the interesting image information is usually contained in the interior of a 3D medical dataset and no deformation occurs at the boundaries of the volume.

In classical FFD approaches (Rueckert et al., 1999), after performing these two steps the new coordinates for every object point are explicitly calculated in the third and the final step based on the shifted coordinates of the control points. To circumvent this computation expense in our algorithm a discrete and uniform grid is considered in parameter space. The function

$$
\mathbf{D}(s, t, u)=\sum_{i=0}^{l} \sum_{j=0}^{m} \sum_{k=0}^{n} B_{i}^{l}(s) B_{j}^{m}(t) B_{k}^{n}(u)\left(b_{i, j, k}+\mathbf{M}\left(b_{i, j, k}\right)\right)
$$


incorporating the displacement function $\mathbf{M}$ is evaluated only on this sparse grid, defining the respective texture coordinates. In order to achieve a better approximation of the original shape of the deformation function the discrete grid can be set denser than the control lattice. After sampling the function $\mathbf{D}$, the resulting texture coordinates on the sparse grid are used to propagate the deformation onto the whole volume using trilinear interpolation. For this purpose, the 3D Bézier function is approximated with a 3D piecewise linear model (Soza, 2005; Hastreiter et al., 2004). This approach is computationally less expensive since there is no need to process the whole 3D image voxel by voxel to obtain new intensity values which is necessary in software approaches. Instead, graphics hardware is employed to reduce the execution time of these expensive operations. A 2D example presenting an approximation of FFD with piecewise linear patches is shown in Figure 2.

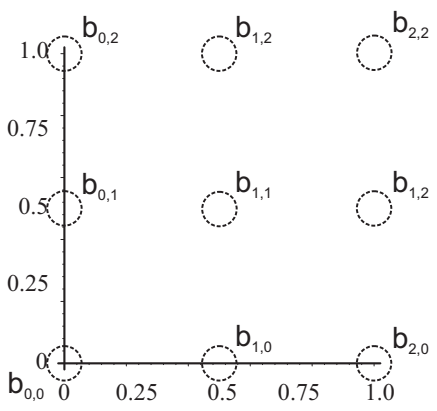

a)

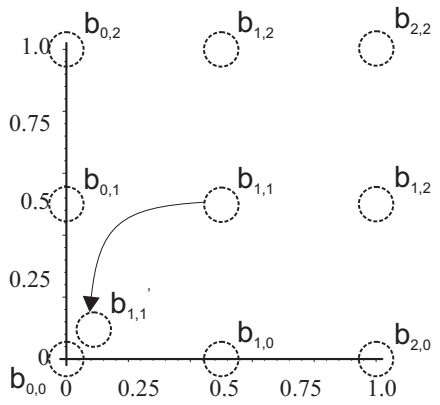

b)

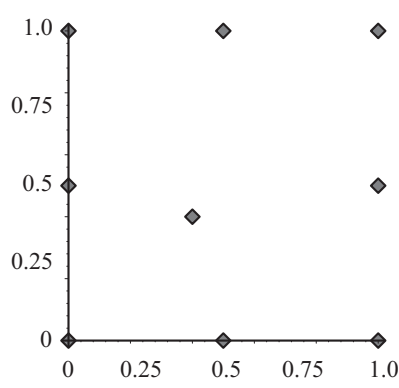

C)

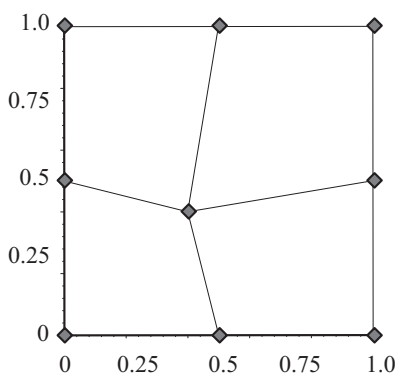

d)

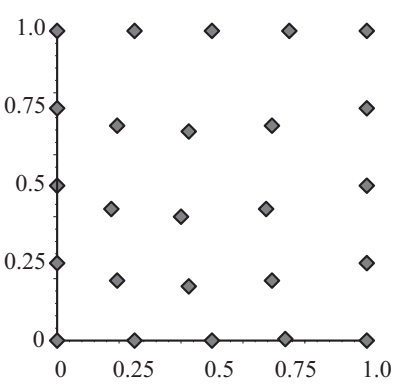

e)

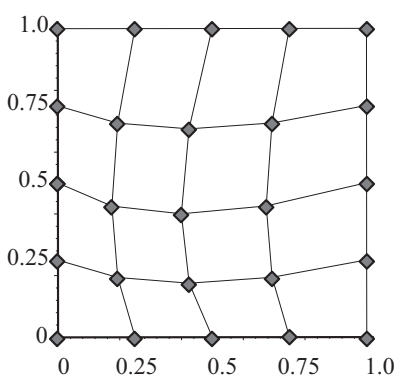

f)

Fig. 2. Subdivision of a slice into 2D piecewise linear patches. a) The Bézier function is defined over a $3 \times 3$ lattice. $b$ ) Control point $b_{1,1}$ was moved from its initial position $(0.5,0.5)$ to $(0.1,0.1)$. This resulted in $\mathbf{D}(0.5,0.5)=(0.4,0.4) . c)$ Values of the image of function $\mathbf{D}$ on a uniform discrete grid $3 \times 3$. d) Resulting $2 \mathrm{D}$ piecewise linear subdivision of the slice. e) Values of the image of function $\mathbf{D}$ on a uniform $5 \times 5$ grid. f) Piecewise linear subdivision of the slice based on the values from $e$ ) 


\subsection{Optimization}

A very efficient optimization strategy is the simultaneous perturbation stochastic approximation (SPSA) (Spall, 1992) method for multivariate optimization problems which was adapted to considerably improve the optimization process. It is an excellent technique for both local and global optimization and is of equal or greater efficiency in terms of overall cost compared to other optimization approaches (Maryak and Chin, 2001; Spall, 2000; Spall et al., 1999). In comparison to Powell's optimization scheme (Press et al., 2002), SPSA further contributes to robustness and efficiency of the registration process due to simultaneous perturbation of the optimized parameters and due to estimated gradient information of the similarity measure.

Let us denote the vectors of the translations $t_{i}$ of the control points in the freeform deformation control lattice by $\boldsymbol{t}_{\boldsymbol{k}}$. At iteration $k+1$, the SPSA procedure updates these free parameters according to:

$$
\boldsymbol{t}_{\boldsymbol{k}+\mathbf{1}}=\boldsymbol{t}_{\boldsymbol{k}}-a_{k} \boldsymbol{g}_{\boldsymbol{k}}\left(\boldsymbol{t}_{\boldsymbol{k}}\right)
$$

Here, $a_{k}$ is a scalar sequence defined later in Equation 7 and $\mathbf{g}_{k}\left(\boldsymbol{t}_{\boldsymbol{k}}\right)$ is the estimate of the gradient of the optimized NMI function for the parameter vector $\boldsymbol{t}_{\boldsymbol{k}}$.

The gradient approximation involves two measurements of the NMI at the perturbed parameter vector $\boldsymbol{t}_{\boldsymbol{k}}$. Specifically, the component $i$ of this gradient is given by

$$
g_{k}^{i}\left(\boldsymbol{t}_{\boldsymbol{k}}\right)=\frac{N M I\left(\boldsymbol{t}_{\boldsymbol{k}}+c_{k} \boldsymbol{\Delta}_{\boldsymbol{k}}\right)-N M I\left(\boldsymbol{t}_{\boldsymbol{k}}-c_{k} \boldsymbol{\Delta}_{\boldsymbol{k}}\right)}{2 c_{k} \Delta_{k}^{i}} .
$$

In this equation, $\Delta_{k}^{i}, i=1, \ldots, n$ are elements of the user-specified perturbation vector $\boldsymbol{\Delta}$. For this perturbation a Bernoulli distribution is used consisting of +1 and -1 both being generated with the same probability of 0.5 , as suggested in (Spall, 1992). $a_{k}$ and $c_{k}$ are sequences depending on the constants $a, A, c, \alpha$ and $\gamma$ and are defined as

$$
\begin{aligned}
a_{k} & =\frac{a}{(A+k)^{\alpha}}, \\
c_{k} & =\frac{c}{k^{\gamma}} .
\end{aligned}
$$

Additionally, these sequences have to fulfill the following conditions to ensure convergence of the iterative procedure:

$$
\alpha-2 \gamma>0,3 \gamma-\frac{\alpha}{2} \geq 0,0<\gamma<\alpha<1
$$


with $a>0, c>0, A \geq 0$. The essential strength of this iterative method is the underlying gradient approximation which requires only two measurements of the objective function independent of the dimension of the parameter domain. In comparison to other optimization approaches such as Powell's direction search method (Press et al., 2002), this leads to a significantly reduced number of iterations during the optimization process. In Figure 3, the convergence processes for Powell and SPSA optimization are compared. In order to obtain a similar value for NMI, i.e. a similar registration quality, SPSA needs significantly less iterations. In comparison to Powell optimization, the number of iterations is reduced to about $15 \%$ (Soza, 2005), resulting in less computational cost.

Powell optimization

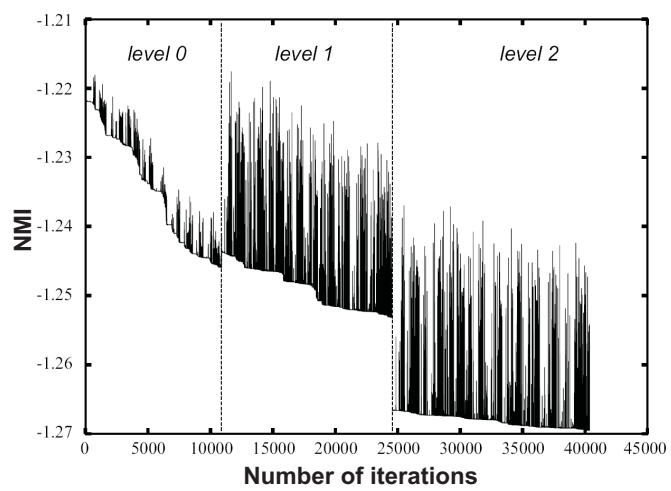

SPSA optimization

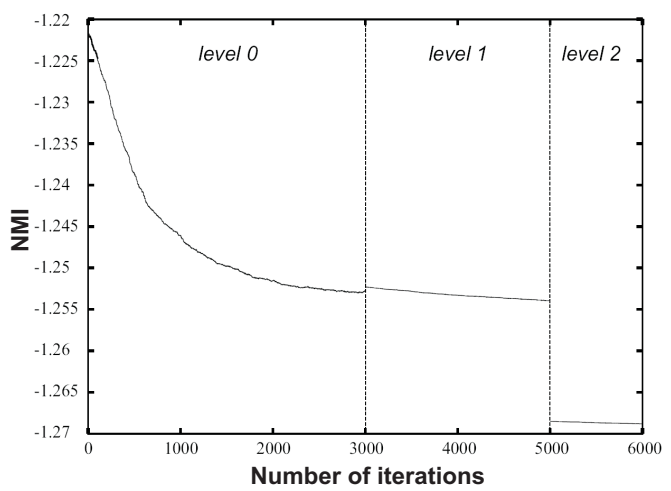

Fig. 3. Comparison of SPSA and Powell optimization. In each plot, the value of the similarity measure in dependence on the iteration number is shown. The different levels of the multiresolution hierarchy are separated by vertical lines.

Moreover, direct measurements of the function are taken for the estimation of the gradients and no explicit gradient information has to be provided for the algorithm. This enables efficient application of this technique to the optimization of normalized mutual information which is dependent on a discrete histogram. Thus, it has an analytically and computationally complex derivative. Since the optimal parameters do not necessarily have to be obtained in the last iteration of the SPSA procedure, an additional third function measurement can be optionally taken in each iteration to obtain the current optimal parameters. However, choosing appropriate configuration parameters for the registration problem results in an almost certain convergence. Therefore, after initial experiments, we performed only two evaluations of the NMI in each iteration which further reduces the computational expense. 


\subsection{Registration framework}

Before performing the non-linear registration of the source and the target dataset using Bézier functions, a rigid registration is computed which is used as an initial estimate (Hastreiter and Ertl, 1998). Thereafter, the source dataset is loaded into texture memory and is embedded in a lattice of control points. At the beginning of the registration, this lattice has the form of a uniform parallelepiped (see Figure 4).

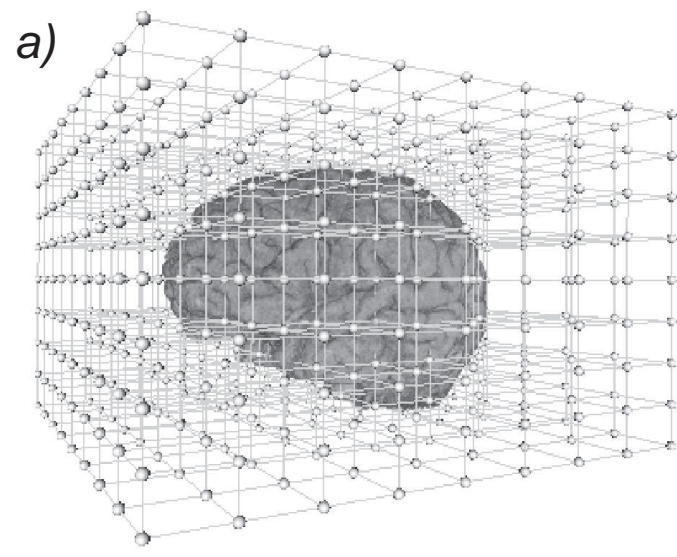

b)

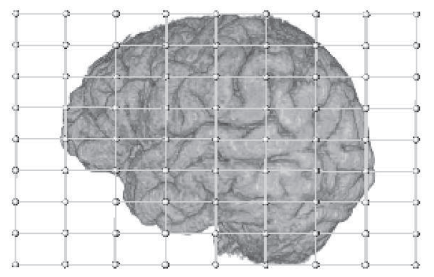

Fig. 4. 3D free-form deformation. A dataset is initially embedded in a uniform lattice of control points: a) 3D view b) Parallel projection

Then, during an iterative procedure, free control points in the lattice are manipulated in such a way that the deformed volume aligns with the target volume. The optimal positions of the control points are estimated with the SPSA algorithm (Section 2.3). The quality of the registration is measured using NMI according to Section 2.1. Thereby, the degrees of freedom are the translation vectors from the initial positions of the inner control points in the lattice. In each iteration, the coordinates of all control points are stochastically perturbed and the new deformed volume is recalculated based on a piecewise linear approximation of the Bézier function. The algorithm optimizes the similarity measure between the recalculated and the target volume until convergence is reached, i.e. the similarity measure does not change significantly over time, or a maximum number of iterations is reached.

\section{$3 \quad$ Experimental setup}

In this section, an overview of the image data used for the experiments is given (Section 3.1). Furthermore, the setup for evaluating the registration framework 
with respect to quality and accuracy is described in Section 3.2. Finally, the data processing and evaluation techniques that are applied for correcting and evaluating susceptibility artifacts in DTI data are provided in Section 3.3. This includes information about the patient population, reconstruction of the pyramidal tract, brain extraction and rigid registration in preparation for nonlinear registration.

\subsection{Image data}

For the reconstruction of fiber tract systems such as the pyramidal tract, DTI data was acquired. For the DTI measurements, a single-shot spin-echo diffusion weighted EPI sequence was used (parameters: $\mathrm{TR}=9200, \mathrm{TE}=86$ $\mathrm{ms}, \mathrm{b}_{\text {high }}=1000 \mathrm{~s} / \mathrm{mm}^{2}, \mathrm{~b}_{\text {low }}=0 \mathrm{~s} / \mathrm{mm}^{2}$, field of view $240 \mathrm{~mm}, 1500 \mathrm{~Hz} / \mathrm{Px}$ bandwidth, average time for 5 measurements: $5 \mathrm{~min}$ and $31 \mathrm{sec})$. For each slice, one reference image without diffusion sensitization (B0) as well as six images with diffusion sensitization in non-collinear directions (B1 - B6) were gathered (gradient directions: $( \pm 1,1,0),( \pm 1,0,1)$, and $(0,1, \pm 1))$. With respect to anatomical information, the $\mathrm{B} 0$ reference image provides the best representation though in poor detail. The other images show diffusion properties. The acquired DTI datasets had a resolution of $128 \times 128 \times 60$ voxels with a respective voxel size of $1.875 \times 1.875 \times 1.9 \mathrm{~mm}^{3}$.

Additionally, an anatomical dataset was acquired for planning and navigation in neurosurgery. Since anatomical MR data shows negligible distortions, it was also used as reference data for compensating susceptibility artifacts of EPI data using non-linear registration. For data acquisition, a 3D gradient echo sequence such as MPRAGE (Magnetization Prepared Rapid Acquisition Gradient Echo) was used (parameters: $\mathrm{TR}=2020 \mathrm{~ms}$, $\mathrm{TE}=4.38 \mathrm{~ms}$, field of view $250 \mathrm{~mm}, 130 \mathrm{~Hz} / \mathrm{Px}$ bandwidth, measurement time $8 \mathrm{~min} 39 \mathrm{sec}$ ). The acquired MPRAGE datasets had a resolution of $512 \times 512 \times 160$ voxels with a respective voxel size of $0.488281 \times 0.488281 \times 1.0 \mathrm{~mm}^{3}$.

All MR imaging was performed using a Magnetom Sonata Maestro Class 1.5 Tesla scanner (Siemens Medical Solutions, Erlangen, Germany) equipped with a gradient system with a field strength of up to $40 \mathrm{mT} / \mathrm{m}$ (effective $69 \mathrm{mT} / \mathrm{m}$ ) and a slew rate of up to $200 \mathrm{~T} / \mathrm{m} / \mathrm{s}$ (effective $346 \mathrm{~T} / \mathrm{m} / \mathrm{s}$ ).

\subsection{Setup for evaluation of registration framework}

The registration framework described in this work was applied to DTI and anatomical MR data in order to correct for the susceptibility artifacts inherent to DTI-EPI data. Since the accuracy of the registration framework is crucial 
for the quality of the correction, a number of initial experiments was performed in order to prove the value of the framework.

In a first experiment, the entire registration framework was evaluated by recovering a known ground truth transformation. For this purpose, an anatomical dataset was warped with a predefined non-linear transformation. The known transformation modeled expansion of the brain in the frontal lobe in order to simulate correction of susceptibility artifacts, which impress the frontal lobe. The original dataset was then registered to the deformed dataset and the resulting transformation was compared to the known transformation.

In addition to numerically comparing the calculated transformation obtained from non-linear registration with the ground truth, difference images between the deformed and the registered dataset were investigated.

Since the registration of DTI data is more delicate due to a limited image resolution, the quality of registration between DTI and anatomical MR data was assessed in a second experiment. For this purpose, distinct landmarks were identified in both the DTI and MPRAGE dataset after non-linear registration and statistical values for the distances between the landmarks were measured.

A supplementary strategy to assess the quality of registration of DTI with anatomical MR data, was visual inspection by a medical expert. For this purpose, a semi-transparent overlay of a DTI data window onto MR slices was used as well as overlays of contours from the anatomical MR scan over the corresponding DTI slice, before and after registration, respectively.

\subsection{Setup for distortion correction and evaluation techniques}

The presented registration approach was used to evaluate the impact of susceptibility distortions on the location of the pyramidal tract (motor tract system) which is monitored during brain surgery by means of functional neuronavigation. This motor tract system has to be spared during surgery in order to avoid post-operative motor deficits. In this context, the potential misalignment due to susceptibility artifacts is of high interest for the surgeon.

In a retrospective study, datasets of 14 patients ( 8 female, 6 male, age range 19-74 years, mean age 44.2 years) were investigated. Among them, 12 patients suffered from gliomas (WHO (World Health Organization) grade I: 1, grade II: 3 , grade III: 6 , grade IV: 2 ) and 2 patients from cavernoma. In each patient, pre-operative anatomical MR as well as DTI based on EPI was measured. During EPI acquisition, gradient emphasis was enabled to leverage the impact of eddy currents as well as lipid suppression for eliminating chemical shift. To compensate for the remaining susceptibility artifacts, the presented 
non-linear registration approach was applied. The distortion-corrected EPI datasets were then used for integration into the MPRAGE data to make fiber tract data available for surgical planning and neuro-navigation.

For each patient, the spatial distortion of the pyramidal tract was analyzed on the hemisphere where the lesion was located. The respective fiber tract bundle was reconstructed using the DTI task card version 1.66 (Magnetic Resonance Center, Massachusetts General Hospital, Boston) on a Siemens workstation using the MR software MRease N4_VA21B under syngo VB10I. This was accomplished using a knowledge-based multiple-ROI (region of interest) approach whereas the tracking algorithm was initiated from user-defined seed regions. Tracking was thereby performed in both retrograde and orthograde direction. A vector step length of $0.5 \mathrm{~mm}$, an angular threshold of 35 degree and a fractional anisotropy threshold of 0.3 were used.

The fiber information was then used to determine all voxels containing fibers within the B0 dataset. Those voxels were marked by setting the respective grey value to a predefined, unambiguous value. After non-linear registration of B0 and MPRAGE data, the derived transformation field was applied to the marked B0 dataset to compensate the susceptibility artifacts. This resulted in a shift of marked voxels in areas where distortions were present in the B0 data.

To prepare the datasets for registration, a segmentation of the brain was performed which in this case was automatically accomplished using the Brain Extraction Tool (Smith, 2002). This is of special importance for intra-operative datasets where the intra-operative head frame is visible as well as artifacts from the metal screws. The fractional intensity threshold, which is an extraction parameter for controling the size of the segmented area, was set to 0.5 (default value) in case of the MPRAGE datasets and was in the range of 0.1 in case of the BO datasets. The quality of the segmentation result was verified for each dataset.

First, the B0 dataset together with the MPRAGE imaging data were rigidly registered. Then, the proposed approach for non-linear registration employing SPSA for optimization was applied. According to the suggestions in (Spall, 1998), the configuration parameters for SPSA optimization were set to $\alpha=$ 0.101 and $\gamma=0.606$. However, experiments showed that small variations of these values have no influence on the quality of the registration result. Furthermore, our own investigations resulted in values of 100.0, 10.0 and 0.5 for the constants $A, a$ and $c$, respectively.

For each pair of the brain images, the FFD registration was performed in a three-stage multiresolution approach. A non-linear registration at a coarse resolution level was performed first. The resulting transformation served as 
input for the second stage at a higher resolution level, and so on. The third stage of the multiresolution approach corresponded to the original resolution of the datasets. With increasing resolution, the registration result is gradually improved on each hierarchy level.

All experiments were conducted with a control lattice consisting of $9 \times 9 \times 9$ control points which has been assessed as adequate for the registration of DTI/MPRAGE data in a series of experiments. In order to better approximate the corresponding Bézier function, the function was sampled on a denser grid of $17 \times 17 \times 17$ points.

All calculations were performed on a PC equipped with an Intel Pentium $4(2.4 \mathrm{GHz})$ and a NVIDIA Quadro4 graphics card (NVIDIA, Santa Clara, CA, USA) with $128 \mathrm{MB}$ graphics memory. The average computation time was $1.5 \mathrm{~h}$. Thereby, $40 \mathrm{~min}$ were required for data conversion, brain extraction, computation of fibers and extraction of voxels containing fibers. The rigid registration providing an initial estimate for the non-linear registration process was in the range of 1-2 minutes. For non-linear registration using hardwareacceleration, a single resampling step and evaluation of NMI for a dataset comprising $512 \times 512 \times 160$ voxels required 0.96 seconds. The overall computing time for non-linear registration was in the range of 50 minutes.

Before non-linear registration, the distortions inherent to DTI data due to susceptibility artifacts result in a displacement of fibers. Since EPI distortions occur in phase-encoding direction (which is the anterior-posterior direction in our case), the measurement of anterior-posterior distortions is of major interest. Accordingly, the distortions in left-right direction are expected to be low. Since MR acquisition is typically performed in slice selection mode, no distortions in $\mathrm{z}$ direction occur.

For each patient, the extent of distortions was evaluated by analyzing corresponding axial slices within the unregistered and the registered B0 dataset. For every second slice containing fibers, the maximum displacement of the fibers was determined, i.e. the offset of the marked voxels. For this purpose, we measured the maximum distance between two corresponding boundary points in left-right and anterior-posterior direction. In Figure 5 the approach for measuring the displacement in axial slices in left-right and anterior-posterior direction is illustrated.

In order to roughly summarize the extent of distortion in the different patients, the numbering of axial slices was adjusted in each dataset providing a crossalignment between different patients. For this purpose, the slice containing the upper boundary of the lateral ventricles was assigned a common slice number in all patients, all other slices in each dataset were renumbered accordingly. As a result, anatomical structures as well as areas of major distortions are 
located in slices with common number allowing averaging of measured results per slice number. In a second test series, we aligned the skull base in each dataset. As a result, most distinct distortions occurred near the cortex and the brainstem.
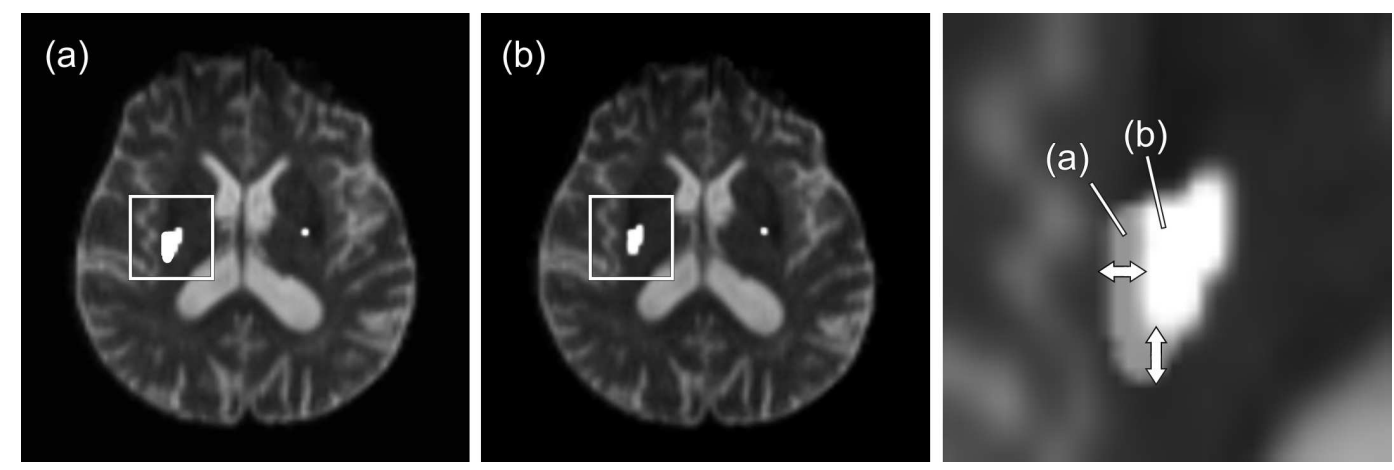

Fig. 5. Evaluation scheme of susceptibility distortions affecting the pyramidal tract in a 68-years-old male patient with a right temporo-mesial WHO grade III anaplastic astrocytoma. The Marked voxels represent the right pyramidal tract within a B0 image before (left) and after (middle) non-linear registration. Transparent overlay analysis (see arrows) was applied in order to measure the maximal displacement in left-right and anterior-posterior direction (right).

\section{Results}

In a first step, the presented algorithm for non-linear registration was evaluated with respect to the obtained registration accuracy using different techniques (Section 4.1). Subsequently, the results of the experimental setup for distortion correction are presented where the extent of distortion encountered for the pyramidal tract due to susceptibility artifacts is evaluated (Section 4.2).

\subsection{Registration accuracy}

In order to verify the quality and accuracy of registration, different techniques were applied. In the first experiment, a known transformation was defined. Since the susceptibility artifacts result in a compression of the frontal lobe, which requires an expansion during non-linear registration, a known transformation expanding the frontal lobe was chosen. The maximum displacement of the parameter values associated to the control points was $50 \mathrm{~mm}$ for the pre-defined deformation. This known transformation was applied to each of the 14 MPRAGE patient datasets. For each patient, the original dataset was 
then non-linearly registered to the deformed one and the resulting transformation was compared to the known transformation. A comparison between the parameter values of the known transformation and the values obtained after non-rigid registration is shown in Table 1 . The average deviation is in the range of $0,18 \mathrm{~mm}$, which indicates a high quality of registration. The maximum deviation from the predefined values is $2,26 \mathrm{~mm}$ (Patient 03), even though the registration result for this patient shows good quality. This can be attributed to a control point that lies outside the region occupied by brain tissue within the volume dataset, having only a small effect on the brain deformation.

\begin{tabular}{|l||r|r|r|}
\hline & $\begin{array}{r}\text { max } \\
{[\mathrm{mm}]}\end{array}$ & $\begin{array}{r}\text { mean } \\
{[\mathrm{mm}]}\end{array}$ & $\begin{array}{r} \pm \mathrm{sd} \\
{[\mathrm{mm}]}\end{array}$ \\
\hline \hline Patient 01 & 0.10775 & 0.09239 & 0.01145 \\
\hline Patient 02 & 0.35325 & 0.09083 & 0.10974 \\
\hline Patient 03 & 2.25950 & 0.29429 & 0.55781 \\
\hline Patient 04 & 2.06150 & 0.21863 & 0.45028 \\
\hline Patient 05 & 0.28850 & 0.07693 & 0.10544 \\
\hline Patient 06 & 1.18525 & 0.18813 & 0.29220 \\
\hline Patient 07 & 1.09575 & 0.16912 & 0.28390 \\
\hline Patient 08 & 0.29925 & 0.07813 & 0.10441 \\
\hline Patient 09 & 1.37000 & 0.19794 & 0.32393 \\
\hline Patient 10 & 1.72000 & 0.18451 & 0.35378 \\
\hline Patient 11 & 1.87650 & 0.23788 & 0.43763 \\
\hline Patient 12 & 2.02675 & 0.23096 & 0.43299 \\
\hline Patient 13 & 1.40675 & 0.19684 & 0.32726 \\
\hline Patient 14 & 0.40675 & 0.11390 & 0.12648 \\
\hline \hline All patients & 2.25950 & 0.17813 & 0.31448 \\
\hline
\end{tabular}

Table 1

Absolute difference between the parameter values obtained from non-linear registration and the parameter values of the known transformation. For each patient, the maximum and the mean value as well as the standard deviation (sd) is given in [mm]. 
The results of the first experiment were visualized using difference images. Figure 6 shows an axial view of dataset 03. The upper row shows the data warped with the known transformation, where the frontal lobe is expanded. The lower row provides a comparison between the dataset warped with the known transformation (left) and the dataset after non-rigid registration (middle). According to the difference image before and after registration (right in both rows), the registration result shows good alignment with the deformed dataset using the known transformation.
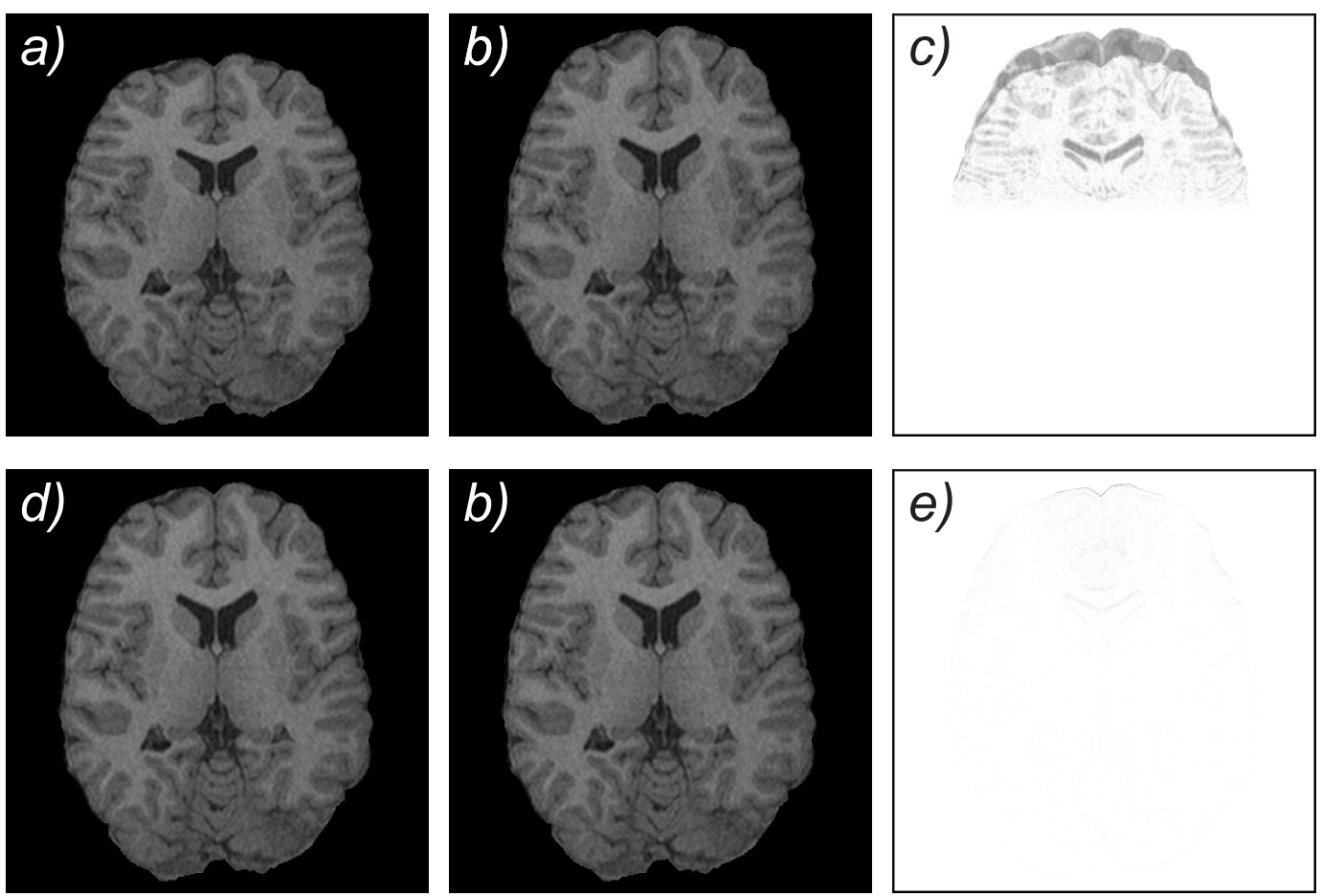

Fig. 6. Corresponding axial slices of an anatomical dataset. Upper row: Original dataset before (a) and after application of known transformation (b) and respective difference image (c). Lower row: Dataset after non-rigid registration $(d)$ in comparison to known transformation (b) and corresponding difference image $(e)$. 
In a second experiment, the quality of registration between DTI and anatomical MR data was investigated. Obviously, since the distortion of the DTI data is not known, it is not possible to investigate the accuracy using a known transformation as before. Instead, an approach based on landmarks is applied. The landmarks were primarily selected in regions where distortions occur such as in the frontal lobe and the brainstem. Thereby, $P_{1}$ and $P_{2}$ denote the most frontal points of both lateral ventricles, $P_{3}$ and $P_{4}$ are positioned at the cerebellum, $P_{5}$ indicates the junction between pons and mid-brain and $P_{6}$ and $P_{7}$ identify the most occipital points of both lateral ventricles.

\begin{tabular}{|l||r|r|r|r|r|r|r|}
\hline & $P_{1}$ & $P_{2}$ & $P_{3}$ & $P_{5}$ & $P_{4}$ & $P_{6}$ & $P_{7}$ \\
\hline \hline Patient 01 & 0.78 & 1.15 & 0.66 & - & 0.94 & 0.76 & 0.61 \\
\hline Patient 02 & 0.81 & - & 0.72 & 0.96 & 2.01 & 0.59 & 0.63 \\
\hline Patient 03 & 1.00 & 1.14 & 0.83 & 1.46 & 1.27 & 0.68 & 0.63 \\
\hline Patient 04 & 1.33 & 0.59 & 0.93 & 0.98 & 2.63 & 0.62 & 0.71 \\
\hline Patient 05 & 1.15 & 1.64 & 1.23 & 0.53 & 2.10 & 0.74 & 0.59 \\
\hline Patient 06 & 1.12 & 0.42 & 0.55 & 0.70 & 0.41 & 0.52 & 0.48 \\
\hline Patient 07 & 0.47 & 0.53 & 1.10 & 0.69 & 1.67 & 0.60 & 0.39 \\
\hline Patient 08 & 0.45 & 0.52 & 0.92 & 0.62 & 1.96 & 0.45 & 0.62 \\
\hline Patient 09 & 0.33 & 0.62 & 0.87 & 0.92 & 2.20 & 0.38 & 0.42 \\
\hline Patient 10 & 1.41 & 1.35 & 0.78 & - & 1.26 & 0.71 & 0.65 \\
\hline Patient 11 & 1.40 & 0.82 & 1.03 & 1.21 & 2.40 & 0.81 & 0.75 \\
\hline Patient 12 & 0.65 & 0.29 & 0.66 & 0.78 & 1.17 & 0.55 & 0.39 \\
\hline Patient 13 & 0.45 & 1.26 & 0.63 & 0.88 & 1.44 & 0.73 & 0.69 \\
\hline Patient 14 & 0.69 & 0.66 & 1.07 & 2.04 & 2.41 & 0.81 & 0.58 \\
\hline \hline mean [mm] & 0.86 & 0.85 & 0.86 & 0.98 & 1.71 & 0.64 & 0.58 \\
\hline sd [mm] & 0.06 & 0.35 & 0.29 & 0.76 & 1.04 & 0.04 & 0.02 \\
\hline
\end{tabular}

Table 2

Quality of non-linear registration for distortion correction in DTI data. For each patient, the distance between distinct anatomical landmarks $P_{1}-P_{7}$ in the DTI and anatomical MR dataset was measured and is given in $[\mathrm{mm}]$. In three cases, no distance could be obtained: In two patients, $P_{5}$ was out of the scan volume, in one patient, $P_{2}$ was infiltrated by the tumor. 
In Table 2, the measurement results are summarized and the mean value \pm standard deviation (sd) is given in [mm]. The misalignment for all landmarks is in the range of $1-2 \mathrm{~mm}$. Taking into account that the voxel resolution of the DTI dataset is in the range of $2 \mathrm{~mm}$ and that the landmarks were placed in regions where distortions due to susceptibility artifacts occur, this is a valuable registration result.

Additionally, the registration result between DTI and anatomical MR data was assessed by visual inspection by a medical expert. A semi-transparent overlay of a DTI data window onto MR slices (Figure 7) as well as contour plots (Figure 8 and 9) were applied for this purpose. Before non-linear registration, the distortions inherent in DTI data due to susceptibility artifacts were clearly visible (Figure 8 and 9). The senior author having more than 15 years experience with MRI, approved a satisfying correspondence in the gyri of the central region, the internal capsule, and the brainstem after registration which further confirms the achieved quality.

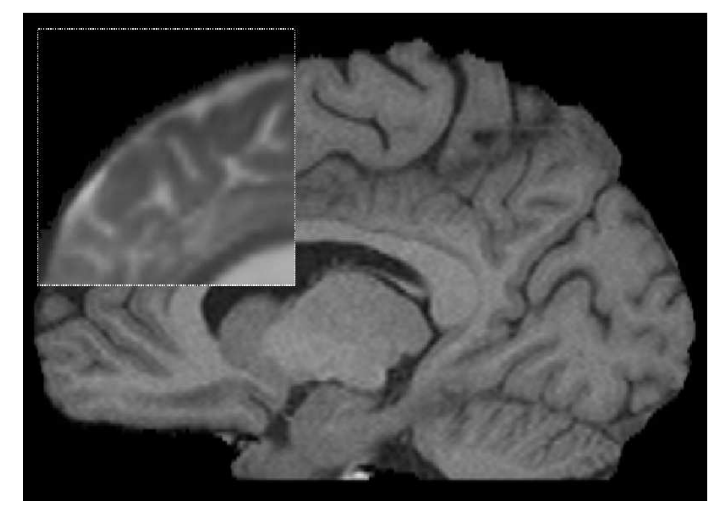

Fig. 7. Illustrative case of a 32-years-old female patient with a recurrent supratentorial primitive neuroectodermal tumor undergoing surgery. Inspection of the registration result using a semi-transparent overlay of DTI data (small data window, movable) and anatomical MR data (background data). 

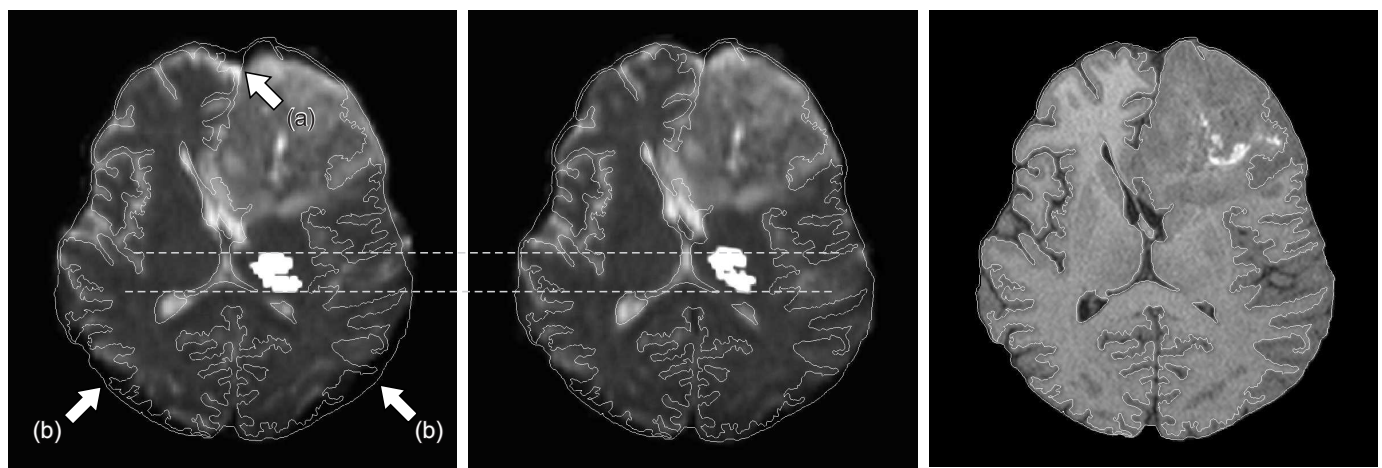

Fig. 8. Illustrative case of a 39-years-old female patient with a right frontal astrocytom (WHO grade III) undergoing surgery. Axial slice of the distorted DT image (B0 image) with the marked pyramidal tract before (left) and after (middle) non-linear registration with contours of MPRAGE slice (right). (a) denotes distortions due to susceptibility artifacts, (b) indicates distortions which can be attributed to the metal screws of the intra-operative head frame. Dashed line allows investigation of displacement.
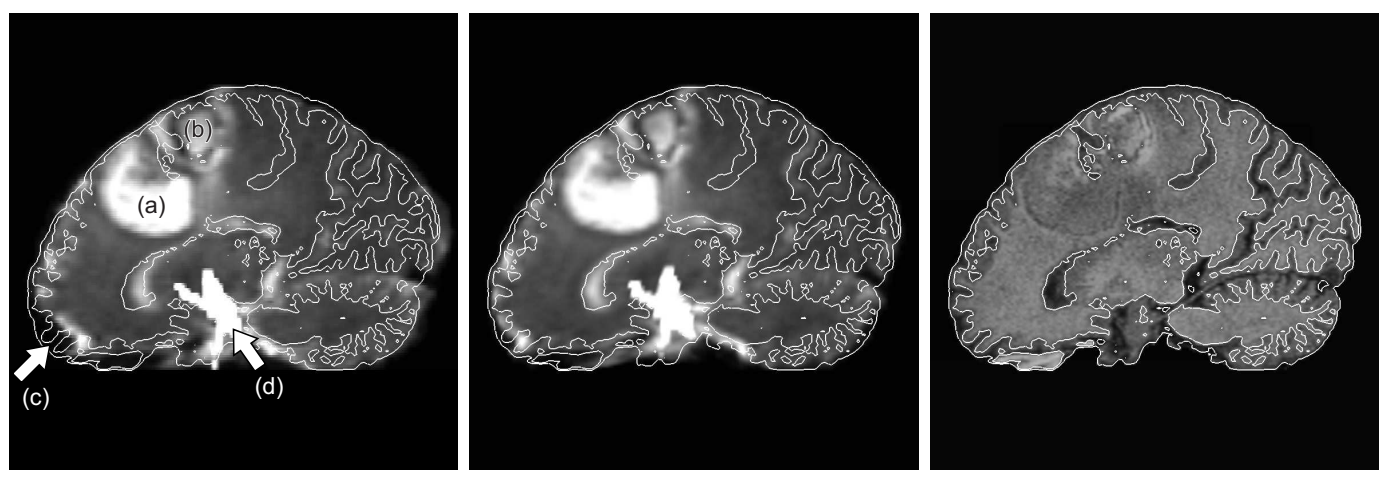

Fig. 9. Illustrative case of a 75-years-old female patient with a right precentral glioblastoma multiforme (WHO grade IV) undergoing surgery. Contours of the anatomical MR slice (right) mapped onto the DT image (B0 image) before (left) and after (middle) non-linear registration support inspection of registration result. (a) denotes an odema, (b) shows the location of the tumor, (c) indicates distortions due to susceptibility artifacts, (d) denotes the pyramidal tract. 


\subsection{Measured susceptibility distortions}

For each patient, the shift of the pyramidal tract after non-linear registration for compensating susceptibility artifacts was measured for each second axial slice. The displacement of the right pyramidal tract in anterior-posterior direction is visualized in Figure 10, where the corresponding measured distortion is plotted for the respective slices.

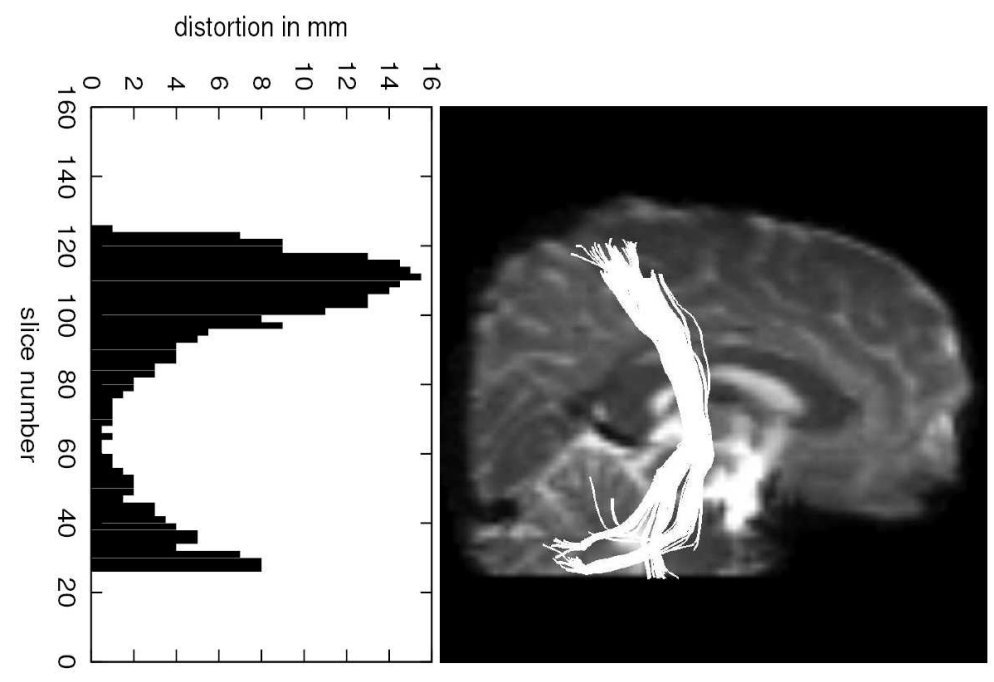

Fig. 10. Measured distortions of the right pyramidal tract in anterior-posterior direction in a 20-years-old female patient with a cavernoma located in the postcentral gyrus. For each second axial slice the distortion of the fibers was measured in corresponding slices of a $\mathrm{B} 0$ dataset before and after registration showing that distinct distortions occurred near the cortex and the brainstem.

The results of all 14 patients are summarized in Figure 11 and 12. In Figure 11 the datasets were aligned according to the upper boundary of the lateral ventricles, in Figure 12 according to the frontal base of the brain. The numbering of the slices was adjusted accordingly. This allows comparing the distortion of DTI data in different individuals to some extent. The displacement of the fibers, i.e. marked voxels, was measured for every second axial slice. In both figures, two separate diagrams indicate the measured distortion in left-right and anterior-posterior direction. For each slice the maximum and minimum measured distortion, as well as the mean value \pm standard deviation of distortion is displayed in the diagram.

The distortion of the pyramidal tract in all patients amounted up to $11.5 \mathrm{~mm}$ in the area of the brainstem with a mean of $3.2 \pm 3.5 \mathrm{~mm}$ in phase encoding direction (anterior-posterior). However, in the area of interest, i.e. around the zone undergoing resection, the mean distortion was limited to $2.4 \pm 1.7 \mathrm{~mm}$ 
with a maximum of $9 \mathrm{~mm}$ in phase-encoding direction. Near the cortex, higher distortions of up to $15 \mathrm{~mm}$ (mean $4.0 \pm 2.8 \mathrm{~mm}$ ) were measured. The left-right distortions amounted up to $9 \mathrm{~mm}$ in the area of the brainstem with a mean of $1.0 \pm 1.5 \mathrm{~mm}$. In the area of interest the mean distortion was even smaller, with a maximum of $7 \mathrm{~mm}$ and a mean of $0.9 \pm 0.9 \mathrm{~mm}$. Near the cortex larger distortions of up to $10 \mathrm{~mm}$ with a mean of $1.3 \pm 1.4 \mathrm{~mm}$ were observed. These results are summarized in Table 3.

\begin{tabular}{|l|c|c|c|c|c|c|}
\hline & \multicolumn{3}{|c|}{ anterior-posterior } & \multicolumn{3}{c|}{ left-right } \\
\cline { 2 - 7 } & $\begin{array}{c}\min \\
{[\mathrm{mm}]}\end{array}$ & $\begin{array}{c}\max \\
{[\mathrm{mm}]}\end{array}$ & $\begin{array}{c}\text { mean } \pm \mathrm{sd} \\
{[\mathrm{mm}]}\end{array}$ & $\begin{array}{c}\min \\
{[\mathrm{mm}]}\end{array}$ & $\begin{array}{c}\max \\
{[\mathrm{mm}]}\end{array}$ & $\begin{array}{c}\text { mean } \pm \mathrm{sd} \\
{[\mathrm{mm}]}\end{array}$ \\
\hline \hline cortex & 0 & 15 & $4.0 \pm 2.8$ & 0 & 10 & $1.3 \pm 1.4$ \\
\hline internal capsule & 0 & 9 & $2.4 \pm 1.7$ & 0 & 7 & $0.9 \pm 0.9$ \\
\hline brainstem & 0.5 & 11.5 & $3.2 \pm 3.5$ & 0 & 9 & $1.0 \pm 1.5$ \\
\hline
\end{tabular}

Table 3

Minimum (min), maximum (max), and mean distortion \pm standard deviation (sd) in anterior-posterior (phase-encoding) and left-right direction measured for different areas of the brain in 14 patients. 

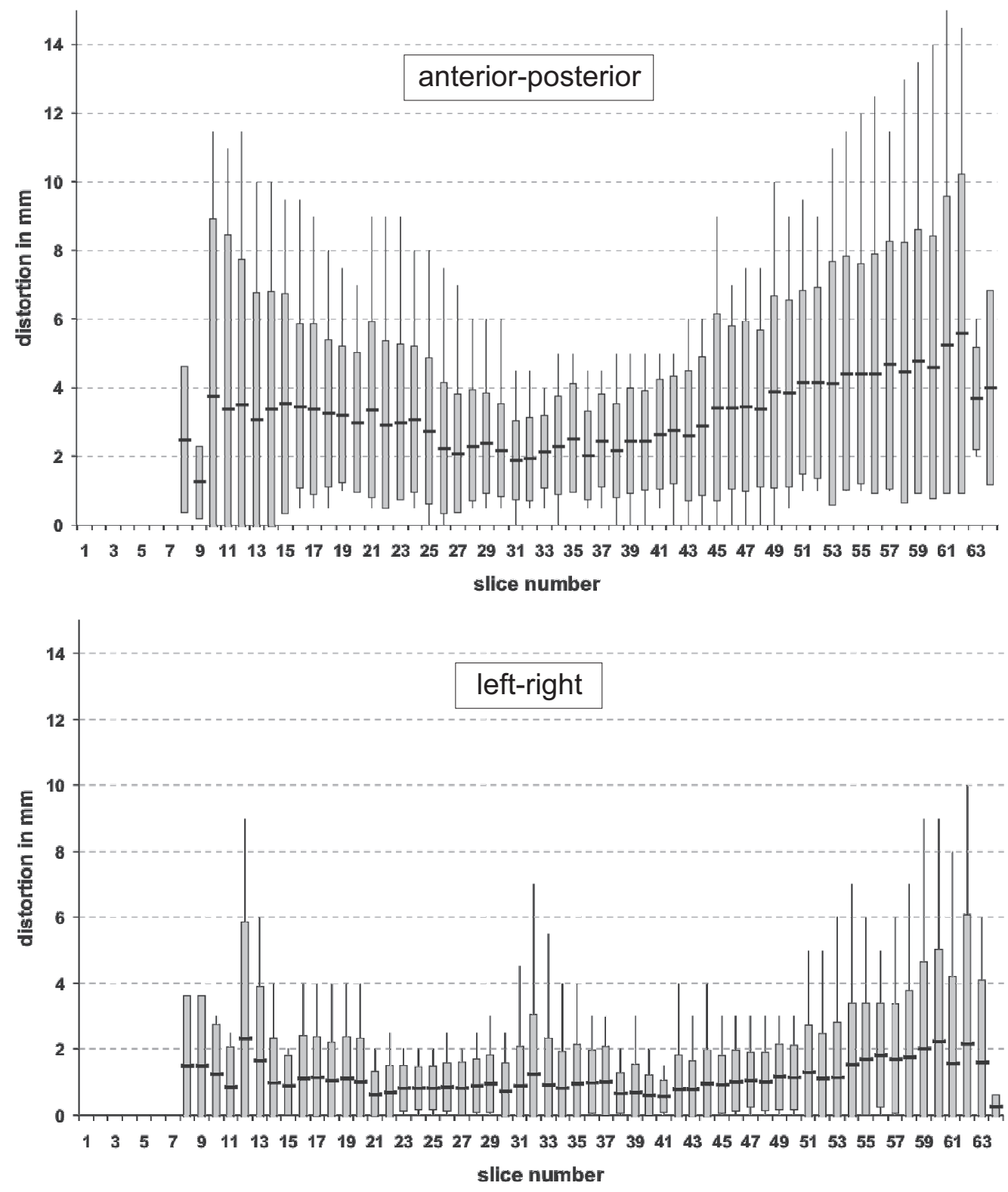

Fig. 11. Datasets aligned according to the upper boundary of the lateral ventricles. Slice numbering starts at the brainstem level. Average distortion \pm standard deviation, as well as minimum and maximum distortions for corresponding axial slices in 14 patients denoted in $\mathrm{mm}$. 

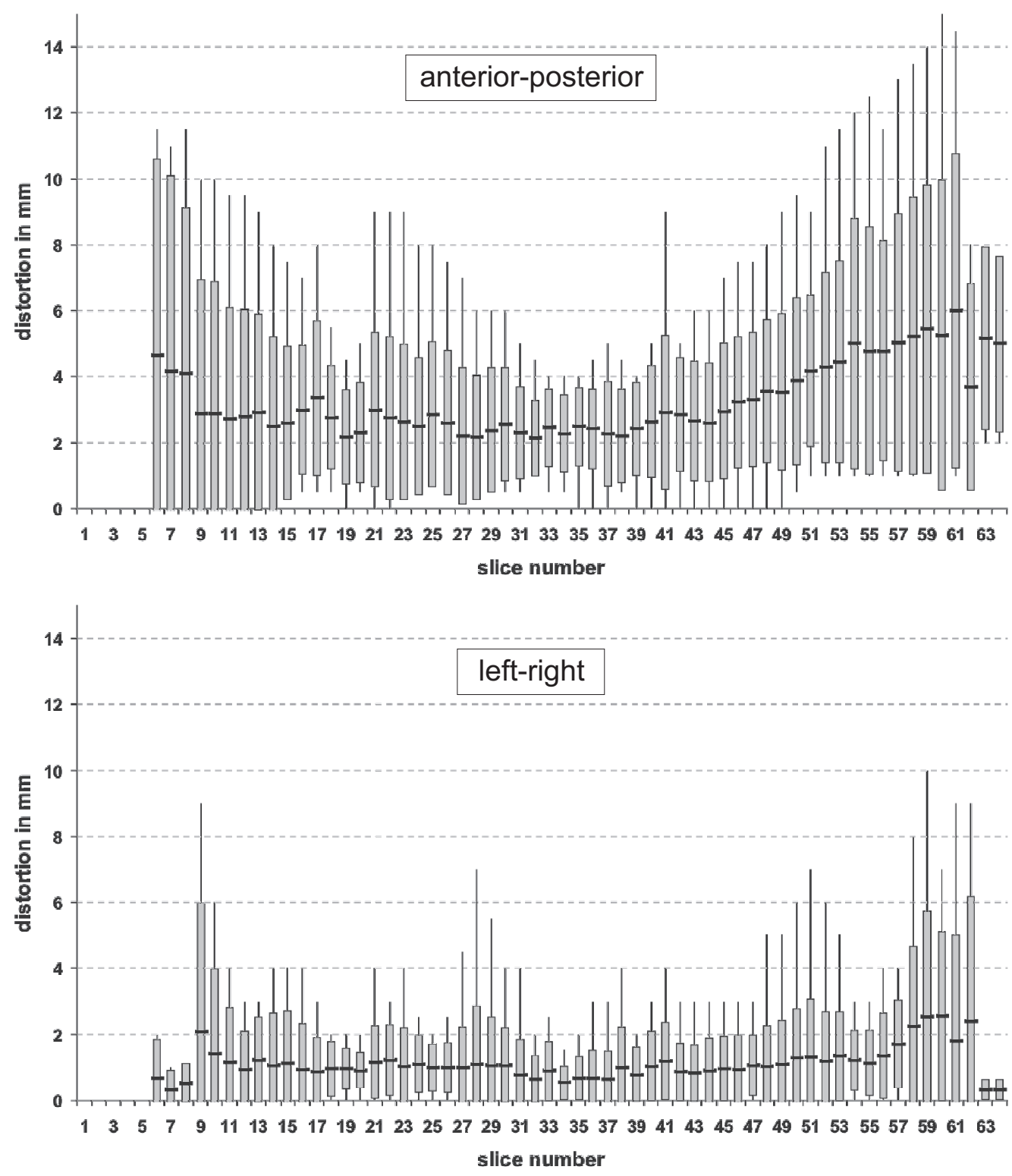

Fig. 12. Datasets aligned according to the frontal base of the brain. Slice numbering starts at the brainstem level. Average distortion \pm standard deviation, as well as minimum and maximum distortions for corresponding axial slices in 14 patients denoted in $\mathrm{mm}$. 


\section{Discussion}

This paper presents an approach for non-linear registration applied for DTI distortion correction extending our previous work (Hastreiter and Ertl, 1998; Hastreiter et al., 2000; Soza et al., 2002; Hastreiter et al., 2004; Soza et al., 2004). In this context, the optimization process was considerably improved using the SPSA optimization strategy. Compared to Powell's direction search method (Press et al., 2002) that we applied up to now, the SPSA method is much better suited to overcome local minima which contributes to the robustness of the registration framework. Furthermore, SPSA is an excellent method for both local and global optimization and has equal or greater efficiency in terms of overall cost compared to other optimization approaches (Maryak and Chin, 2001; Spall, 2000; Spall et al., 1999). For an equal registration accuracy expressed by similar NMI values, the number of iterations required by SPSA is reduced to about 15 \% (Soza, 2005) compared to Powell optimization. Another efficiency aspect of SPSA is its behavior for an increased number of control points. As an advantage, the number of iterations does not scale linearly with the number of control points, as encountered in case of the Powell optimizer. Therefore, the computing time for non-linear registration was only about 50 minutes in spite of a considerably increased number of free control points which is about 13 times higher in the employed $9 \times 9 \times 9$ lattice $\left(7^{3}\right.$ free control points) compared to the $5 \times 5 \times 5$ lattice (Soza et al., 2002) $\left(3^{3}\right.$ free control points).

In addition to our hardware-based strategy of non-linear registration, SPSA further contributes to the robustness and efficiency of the registration approach due to simultaneous perturbation of the optimized parameters and due to estimated gradient information of the similarity measure.

The presented approach for non-linear registration was used to correct for susceptibility artifacts inherent to EPI data in the context of DTI. Within an iterative procedure, a displacement field is computed resulting in a shift of fibers represented by marked voxels. This indirect approach relying on precomputed fibers is necessary since applying the deformation field to the B1-B6 data would not provide correct tensors due to the geometric nature of the tensor information. This is related to the warping of the DTI data requiring a re-orientation of the respective tensors for full correction. Using the presented approach, the difficult problem of tensor re-orientation was elegantly overcome. A severe drawback of approaches currently available for tensor reorientation (Guimond et al., 2002; Ruiz-Alzola et al., 2002; Sierra, 2001) is the significantly increased computational cost due to their algorithmic complexity. In addition to that, these techniques have not yet been fully quantified and theoretically proven, for this reason re-orientation of tensors is circumvented in our registration framework. 
To quantify the extent of distortions resulting from susceptibility artifacts, the presented approach was evaluated for 14 patients. Thereby, the displacement after distortion correction of the pyramidal tract was measured in the hemisphere where the lesion was located. The results were consistent with our expectations showing distinct distortions at the brain surface (in the central region) and near the brainstem. Accordingly, the distortion in the region of the internal capsule was less significant. In general, the main displacement occurred in anterior-posterior direction, i.e. in phase-encoding direction, whereas the left-right displacements proved to be less dominant. This observation is of clinical importance in cases where a tumor causes a medial shift of the pyramidal tract since the position in left-right direction is relatively exactly depicted by DTI data even without non-linear registration. The two approaches for roughly aligning the different patient data to summarize the measured data, using the position of either the lateral ventricles or the frontal brain base as reference, showed no prominent difference regarding the averaged distortion measurements. In spite of the general tendencies, we observed differences between individual patients. For this reason, distortion correction based on non-linear registration has to be applied for each patient dataset separately in order to obtain precise results. This is of special importance for patients where the lesion is close to the pyramidal tract in order to prevent injury of this tract system due to a wrong anticipated position resulting from susceptibility distortions. Nevertheless, the comparison and evaluation involving all datasets shows that susceptibility artifacts are most prominent near the cortex and in the brain stem and affect the position of the pyramidal tract primarily in anterior-posterior direction.

Even though our clinical evaluation is purely retrospective, the presented approach enables for distortion correction of susceptibility artifacts which is utilizable for pre-operative planning. Thereby, eloquent cortical brain areas and major fiber connections such as the pyramidal tract are determined and considered to minimize the risk of post-operative neurological deficits. For this purpose, data from fMRI or MEG identifying eloquent cortical brain areas, are integrated into datasets for stereotactic guidance resulting in so-called functional neuro-navigation (Ganslandt et al., 2004; Nimsky et al., 1999; Nimsky et al., 2004a). The next logical step is to also integrate major fiber connections such as the pyramidal tract computed by tractography algorithms in a stereotactic setup (Kamada et al., 2005; Kinoshita et al., 2005; Clark et al., 2003; Coenen et al., 2001) which requires techniques for distortion correction.

Apart from the registration approach presented in this paper, other strategies exist to account for image distortions inherent to DTI data: In modern functional scanning tools, field maps are implemented to identify and mark areas where the image information can no longer be trusted. However, a correction of image distortions is not provided with this technique. Another strategy are DTI sequences such as diffusion-weighted single-shot STEAM MR imag- 
ing (Nolte et al., 2000) combining a diffusion-weighted spin-echo preparation period and a high-speed stimulated echo acquisition mode (STEAM) MRI sequence, resulting in significantly less imaging distortions.

Essentially, this MRI technique is insensitive to susceptibility gradients, magnetic field inhomogeneities, and chemical shifts (Nolte et al., 2000). However, it suffers from several drawbacks such as a coarser resolution, a lower signal to noise ratio and an increased measurement time which still render its clinical use problematic. For these reasons, EPI remains essential for clinical DTI, requiring strategies for distortion correction of the underlying EPI data.

The presented approach enables correction of susceptibility artifacts in DTI data using non-linear registration and to use this data for neuro-navigation to visualize the correct spatial relation of a tumor with respect to the pyramidal tract. Limitations of the presented approach relate to brain shift effects or tumor removal requiring an update of the navigation with intra-operative image and fiber tract data (Nimsky et al., 2004b; Nimsky et al., 2005) to compensate for these effects (Nimsky et al., 2000). In spite of acceleration based on graphics hardware and a highly efficient optimization strategy, the non-linear registration approach for 3D image data is still too time consuming for intra-operative use. However, the problem of updating the navigation after brain shift or tissue removal also holds for fMRI or MEG data which have to be acquired pre-operatively. In current research, this problem is addressed by simulation approaches aiming at prediction of intra-operative deformations (Soza et al., 2004). The simultaneous application of subcortical electrical stimulation may be another possibility of additional help in the clinical setting. Nevertheless, transient neurological deficits of up to $37 \%$, decreasing to $7 \%$ permanent deficits remain when electrical stimulation is applied alone (Keles et al., 2004). These high numbers emphasize the need for reliable integration of major white matter tracts into neuro-navigation for intra-operative visualization. Our nonlinear registration approach helps to correct for distortions due to susceptibility artifacts and thus supports pre-operative planning in neurosurgery, aiming at integrating major white matter tracts into stereotactic guidance systems.

\section{Conclusions and future work}

Susceptibility artifacts inherent to EPI data cause a spatial distortion of fiber tracts obtained by DTI-based fiber tracking. In order to compensate for these distortions, a non-linear registration approach based on graphics hardware incorporating a highly efficient and robust optimization strategy was proposed. In a first evaluation, the quality of registration provided by the non-linear registration framework was verified. The framework was then applied in a clinical study comprising 14 patients, where distinct distortions were mea- 
sured near the brainstem and at the cortical surface, especially of the frontal lobe. However, we observed a certain variability between different individuals which requires considering each case separately. Correcting the error due to image distortion improves spatially correct pre-operative neurosurgical planning and pre-operative integration of tractography data into neuro-navigation. The presented approach thus contributes to a safer resection in neurosurgery.

In the future, we will focus on performance improvements by exploiting new features of upcoming graphics hardware. Further speed-up could also result from a lattice with control points positioned at specific landmarks or features for selective correction.

\section{Acknowledgments}

This work was supported by the Deutsche Forschungsgemeinschaft in the context of SFB 603 (Project C9) and the Graduate Research Center '3D Image Analysis and Synthesis'. We are especially grateful to A. G. Sorensen (Department of Radiology/Nuclear Magnetic Resonance Center, Massachusetts General Hospital, Boston, USA) for providing the DTI task card software we used for the computation of the fiber tracts.

\section{References}

Basser, P., Pierpaoli, C. 1996. Microstructural and Physiological Features of Tissues Elucidated by Quantitative-Diffusion-Tensor MRI. J Magn Reson Imaging, 111, 209-219.

Basser, P., Mattiello, J., LeBihan, D. 1994. MR Diffusion Tensor Spectroscopy and Imaging. Biophys J, 66(1), 259-267.

Basser, P., Pajevic, S., Pierpaoli, C., Duda, J., Aldroubi, A. 2000. In Vivo Fiber Tractography Using DT-MRI Data. Magn Reson Med, 44, 625-632.

Bodammer, N., Kaufmann, J., Kanowski, M., Tempelmann, C. 2004. Eddy Current Correction in Diffusion-Weighted Imaging using Pairs of Images Acquired with Opposite Diffusion Gradient Polarity. Magn Reson Med, 51(1), 188-193.

Clark, C., Barrick, T., Murphy, M., Bell, B. 2003. White Matter Fiber Tracking in Patients With Space-Occupying Lesions of the Brain: A New Technique for Neurosurgical Planning? Neuroimage, 20(3), 1601-1608.

Coenen, V., Krings, T., Mayfrank, L., Polin, R., Reinges, M., Thron, A., Gilsbach, J. 2001. Three-dimensional Visualization of the Pyramidal Tract in a Neuronavigation System During Brain Tumor Surgery: First Experiences and Technical Note. Neurosurgery, 49(1), 86-92. 
Collignon, A., Vandermeulen, D., Suetens, P., Marchal, G. 1995. Automated Multi-Modality Image Registration Based on Information Theory. Information Processing in Medical Imaging, 3, 263-274.

Ganslandt, O., Buchfelder, M., Hastreiter, P., Grummich, P., Fahlbusch, R., Nimsky, C. 2004. Magnetic Source Imaging Supports Clinical Decision Making in Glioma Patients. Clin Neurol Neurosurg, 107(1), 20-26.

Guimond, A., Guttmann, C., Warfield, S., Westin, C.-F. 2002. Deformable Registration of DT-MRI Data Based on Transformation Invariant Tensor Characteristics. In: Proc. of the IEEE International Symposium on Biomedical Imaging (ISBI).

Hastreiter, P., Ertl, T. 1998. Integrated Registration and Visualization of Medical Image Data. Pages 78-85 of: Proc. CGI.

Hastreiter, P., Rezk-Salama, C., Nimsky, C., Lürig, C., Greiner, G., Ertl, T. 2000. Registration Techniques for the Analysis of the Brain Shift in Neurosurgery. Computers \& Graphics, 24(3), 385-389.

Hastreiter, P., Rezk-Salama, C., Soza, G., Bauer, M., Greiner, G., Fahlbusch, R., Ganslandt, O., Nimsky, C. 2004. Strategies for Brain Shift Evaluation. Med Image Anal, 8(4), 447-464.

Hellier, P., Barillot, C. 2000. Multimodal Non-rigid Warping for Correction of Distortions in Functional MRI. Pages 512-520 of: Proc. MICCAI. Lect. Notes in Comp. Sc. Springer.

Jezzard, P., Clare, S. 1999. Sources of Distortion in Functional MRI Data. Hum Brain Mapp, 8(2-3), 80-85.

Kamada, K., Todo, T., Masutani, Y., Aoki, S., Ino, K., Takano, T., Kirino, T., Kawahara, N., Morita, A. 2005. Combined Use of TractographyIntegrated Functional Neuronavigation and Direct Fiber Stimulation. J Neurosurg, 102(4), 664-672.

Keles, G., Lundin, D., Lamborn, K., Chang, E., Ojemann, G., Berger, M. 2004. Intraoperative Subcortical Stimulation Mapping for Hemispherical Perirolandic Gliomas Located Within or Adjacent to the Descending Motor Pathways: Evaluation of Morbidity and Assessment of Functional Outcome in 294 Patients. J Neurosurg, 100(3), 369-375.

Kindlmann, G. 2004. Superquadric Tensor Glyphs. Pages 147-154 of: Proc. IEEE Symposium on Visualization. Eurographics Association.

Kinoshita, M., Yamada, K., Hashimoto, N., Kato, A., Izumoto, S., Baba, T., Maruno, M., Nishimura, T., Yoshimine, T. 2005. Fiber-Tracking does not Accurately Estimate Size of Fiber Bundle in Pathological Condition: Initial Neurosurgical Experience Using Neuronavigation and Subcortical White Matter Stimulation. Neuroimage, 25(2), 424-429.

Kober, H., Nimsky, C., Möller, M., Hastreiter, P., Fahlbusch, R., Ganslandt, O. 2001. Correlation of Sensorimotor Activation with Functional Magnetic Resonance Imaging and Magnetoencephalography in Presurgical Functional Imaging: A Spatial Analysis. NeuroImage, 14(5), 1214-1228.

Kybic, J., Thévenaz, P., Nirkko, A., Unser, M. 2000. Unwarping of Unidirectionally Distorted EPI Images. IEEE Trans. Med. Imaging, 19(2), 
80-93.

LeBihan, D., Mangin, J.-F., Poupon, C., Clark, C., Pappata, S., Molko, N., Chabriat, H. 2001. Diffusion Tensor Imaging: Concepts and Applications. Journal of Magnetic Resonance Imaging, 13, 534-546.

Mangin, J., Poupon, C., Clark, C., Bihan, D. Le, Bloch, I. 2003. Distortion Correction and Robust Tensor Estimation for MR Diffusion Imaging. Med Image Anal, 6(3), 191-198.

Maryak, J., Chin, D. 2001. Global Random Optimization by Simultaneous Perturbation Stochastic Approximation. Pages 756-762 of: Proc. of the American Control Conference Arlington.

Merhof, D., Hastreiter, P., Soza, G., Stamminger, M., Nimsky, C. 2004. Nonlinear Integration of DTI-based Fiber Tracts into Standard 3D MR Data. Pages 371-37r of: Girod, B., Magnor, M., Seidel, H.-P. (eds), Proc. Vision, Modeling and Visualization (VMV). Akademische Verlagsgesellschaft AKA, Berlin.

Meyer, C., Pauly, J., Macovski, A., Nishimura, D. 1990. Simultaneous Sspatial and Spectral Selective Excitation. Magn Reson Med, 15(2), 287-304.

Mori, S., van Zijl, P. 2002. Fiber Tracking: Principles and Strategies - A Technical Review. NMR Biomed, 15, 468-480.

Mori, S., Crain, B., Chacko, V., van Zijl, P. 1999. Three-Dimensional Tracking of Axonal Projections in the Brain by Magnetic Resonance Imaging. Ann Neurol, 45(2), 265-269.

Nimsky, C., Ganslandt, O., Kober, H., Möller, M., Ulmer, S., Tomandl, B., Fahlbusch, R. 1999. Integration of Functional Magnetic Resonance Imaging Supported by Magnetoencephalography in Functional Neuronavigation. Neurosurgery, 44, 1249-1256.

Nimsky, C., Ganslandt, O., Cerny, S., Hastreiter, P., Greiner, G., Fahlbusch, R. 2000. Quantification of, Visualization of, and Compenstation for Brain Shift by Intraoperative Magnetic Resonance Imaging. Neurosurgery, 47(5), 1070-1080.

Nimsky, C., Ganslandt, O., Fahlbusch, R. 2004a. Functional Neuronavigation and Intraoperative MRI. Adv Tech Stand Neurosurg, 29, 229-263.

Nimsky, C., Ganslandt, O., Hastreiter, P., Wang, R., Benner, T., Sorensen, A., Fahlbusch, R. 2004b. Intraoperative Diffusion-Tensor MR Imaging: Shifting of White Matter Tracts during Neurosurgical Procedures - Initial Experience. Radiology, 234(1), 218-225.

Nimsky, C., Ganslandt, O., Hastreiter, P., Wang, R., Benner, T., Sorensen, A., Fahlbusch, R. 2005. Preoperative and Intraoperative Diffusion Tensor Imaging-based Fiber Tracking in Glioma Surgery. Neurosurgery, 56(1), 130-138.

Nolte, U., Finsterbusch, J., Frahm, J. 2000. Rapid Isotropic Diffusion Mapping Without Susceptibility Artifacts: Whole Brain Studies Using DiffusionWeighted Single-Shot STEAM MR Imaging. Magn Reson Med, 44(5), 731-736.

Press, W., Teukolsky, S., Vetterling, W., Flannery, B. 2002. Numerical Recipes 
in $C++$. Cambridge University, New York.

Roche, A., Malandain, G., Pennec, X., Ayache, N. 1998. The Correlation Ratio as a New Similarity Measure for Multimodal Image Registration. Pages 1115-1124 of: Proc. MICCAI. Lect. Notes in Comp. Sc. Springer.

Rohde, G., Barnett, A., Basser, P., Marenco, S., Pierpaoli, C. 2004. Comprehensive Approach for Correction of Motion and Distortion in DiffusionWeighted MRI. Magn Reson Med, 51(1), 103-114.

Rueckert, D., Sonoda, L., C. Hayes, D. Hill, Leach, M., Hawkes, D. 1999. Nonrigid Registration Using Free-Form Deformations: Application to Breast MR Images. IEEE Trans Med Img, 18(8), 712-721.

Ruiz-Alzola, J., Westin, C.-F., Warfield, S., Alberola, C., Maier, S., Kikinis, R. 2002. Nonrigid Registration of 3D Tensor Medical Data. Med Image Anal, 6(2), 143-161.

Shannon, C., Weaver, W. 1949. The Mathematical Theory of Communication. University of Illinois Press.

Shen, Y., Larkman, D., Counsell, S., Pu, I., Edwards, D., Hajnal, J. 2004. Correction of High-Order Eddy Current Induced Geometric Distortion in Diffusion-Weighted Echo-Planar Images. Magn Reson Med, 52(5), 11841189.

Sierra, R. 2001. Nonrigid Registration of Diffusion Tensor Images. M.Phil. thesis, Swiss Federal Institute of Technology Zurich. SPL Technical Report No. 207.

Smith, S. 2002. Fast Robust Automated Brain Extraction. Hum Brain Mapp, 17(3), 143-55.

Soza, G. 2005. Registration and Simulation for the Analysis of the Intraoperative Brain Shift. Ph.D. thesis, Computer Graphics Group, Computer Science Department, University of Erlangen-Nuremberg.

Soza, G., Bauer, M., Hastreiter, P., Nimsky, Ch., Greiner, G. 2002. Non-rigid Registration with Use of Hardware-Based 3D Bézier Functions. Pages 549-556 of: Proc. MICCAI. Lect. Notes in Comp. Sc., vol. 2489. Springer.

Soza, G., Grosso, R., Nimsky, C., Greiner, G., Hastreiter, P. 2004. Estimating Mechanical Brain Tissue Properties with Simulation and Registration. Pages 276-283 of: MICCAI. Lect. Notes in Comp. Sc., vol. 3217. Springer.

Spall, J. 1998. Implementation of the Simultaneous Perturbation Algorithm for Stochastic Optimization. IEEE T Aero Elec Sys, 34(3), 817-823.

Spall, J. 2000. Adaptive Stochastic Approximation by the Simultaneous Perturbation Method. IEEE Transactions on Automatic Control, 45, 18391853.

Spall, J., Hill, S., Stark, D. 1999. Theoretical Comparisons of Evolutionary Computation and Other Optimization Approaches. Pages 1398- 1405 of: Proc. of the Congress on Evolutionary Computation.

Spall, J. C. 1992. Multivariate Stochastic Approximation Using a Simultaneous Perturbation Gradient Approximation. IEEE Trans Autom Control, 37(3), 332-341.

Studholme, C., Hill, D., Hawkes, D. 1996. Automated 3D Registration of 
Truncated MR and CT Images of the Head. Pages 27-37 of: Proc. Brit. Mach. Vis. Conf. (BMVC).

Studholme, C., Hill, D., Hawkes, D. 1999. An Overlap Invariant Entropy Measure of 3D Medical Image Alignment. Pattern Recogn, 32(1), 71-86.

Weisskoff, R. 1990. Improved Hard-Pulse Sequences for Frequency-Selective Presaturation in Magnetic Resonance. J Magn Reson, 86(1), 170-175.

Wells, W., Viola, P., Kikinis, R. 1995. Multi-modal Volume Registration by Maximization of Mutual Information. Proc. Med. Robot. and Comput Assis. Surg., 55-62.

Wells, W., Viola, P., Atsumi, H., Nakajima, S., Kikinis, R. 1997. Multi-modal Volume Registration by Maximization of Mutual Information. Med Image Anal, 1(1), 35-51.

Westin, C., Maier, S., Mamata, H., Nabavi, A., Jolesz, F., Kikinis, R. 2002. Processing and visualization for diffusion tensor MRI. Med Image Anal, 6(2), 93-108. 